\title{
Cost Sharing with Network Coding in Two-Way Relay Networks
}

\author{
Ertugrul Necdet Ciftcioglu†, Yalin Evren Sagduyuł, Randall Berry $\ddagger$ Aylin Yener $\dagger$ \\ $\dagger$ Dept. of Electrical Engineering \\ The Pennsylvania State University \\ University Park, PA 16802, USA \\ $\ddagger$ Dept. of Electrical Engineering and Computer Science \\ Northwestern University \\ Evanston, IL 60208, USA \\ E-mail: enc118@psu.edu,y-sagduyu@northwestern.edu, rberry@eecs.northwestern.edu, yener@ee.psu.edu
}

\begin{abstract}
We consider a scenario in which two sources exchange stochastically varying traffic with the aid of a bidirectional relay that may perform network coding over the incoming packets. Each relay use incurs a unit cost, e.g., transmission energy. This cost is shared between the sources when packets from both are transmitted via network coding; if traffic from a single source is sent, the cost is passed on to only that source. We study transmission policies which trade-off the average cost with the average packet delay. First, we analyze the cost-delay trade-off for a centralized control scheme using Lyapunov stability arguments. We then consider a distributed control scheme, where each source selfishly optimizes its own cost-delay trade-off by playing a non-cooperative game. We determine the Nash equilibrium and show that it performs worse than the centralized algorithm. However, appropriate pricing at the relay achieves the centralized performance. These algorithms require full information of queue backlogs. Next, we relax this assumption and any source makes the transmission decision depending on whether the other sources queue backlog exceeds a threshold, or not. This needs only one bit information exchange and leads to asymptotically optimal cost, as the delay grows. Finally, we consider cost sharing with only local queue information at each source. The results illustrate new cost-delay trade-offs based on different levels of cooperation and queue information availability.
\end{abstract}

\section{INTRODUCTION}

A simple, yet fundamental model for a multi-hop wireless networks is the two-way relay network shown in Fig. 1. In this network, two sources communicate with each other with the assistance of a single relay node. It has become well established that network coding [1] can improve the throughput of such a two-way relay over traditional storeand-forward routing, e.g. see [2], [3]. In many cases, the use of a relay node will incur a cost, for example representing the energy consumption of the relay. The use of network coding can also reduce this cost [4], [5]. For example, with network coding a single transmission by the relay can be used to forward one packet from each source simultaneously, while with plain routing this would require two transmissions. If the cost per transmission was the same in both cases, then network coding will result in a 50\% reduction in the total cost. However, when the sources have bursty traffic, this cost reduction may come at the price of higher delays, since each source must wait for packets to arrive at the other source in order to exploit the network coding gain. This cost-delay trade-off has been observed in [6], [7] and is the main focus of this paper. Specifically, we consider the design of transmission policies at the sources which trade-off the average cost with the average delay.

We begin by considering centralized policies in which a centralized controller specifies the transmission rate of each source based on perfect information of both source queue states. We use Lyapunov stability arguments as in [8]-[10] to develop rate allocation policies and analyze their cost-delay trade-off. We then consider a model in which each source individually decides its own rate based on the joint queue size, resulting in a non-cooperative game. We characterize the equilibria of this game and show that in general it leads to worse performance than the centralized solution. These equilibria require each source to know the queue size of both sources. We next consider algorithms which do not require full knowledge of the queue sizes at both sources. Specifically, we derive strategies against the worst and best response of the opponent thereby limiting the backlog information to the individual queues only. This leads to the result that appropriate pricing by the relay for the worst-case response can achieve the cost-optimal operation at the expense of increasing the complexity of rate allocation.

Next, we present a simple transmission scheme that requires only one bit information of the other source's queue backlog. The transmission decisions are based on whether the individual queue backlogs exceed a fixed threshold, or not. In particular, we show that it is possible to achieve asymptotically optimal cost, as the packet delay grows with increasing threshold values. Then, we further relax the assumption of queue state knowledge and extend the analysis to the case where sources do not share any information on each other's queue backlog. All of the cost-sharing algorithms proposed in this paper do not require any a priori information of packet arrival statistics and they can operate only with the instantaneous values of queue backlogs.

In terms of related work, we note that there have been a number of works studying the interaction of network coding with stochastically varying traffic in both two-way relay networks [11], [12] and other network topologies [6], [13][15]. There have also been a number of papers studying the trade-off of for wireless networks without network coding, including [9], [10], [16]. Other game theoretic models for network coding have been studied in [17], [18]; in these models the underlying conflict of interest is due flow optimization rather than cost sharing. 


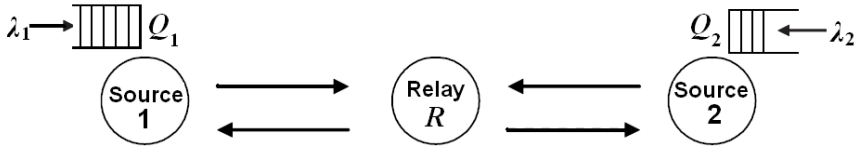

Fig. 1. Two-way relay network: Two sources with packet arrivals and a single relay to exchange the incoming packets.

\section{SySTEM MODEL}

We consider the two-way relay network with two sources 1 and 2, and one relay $R$, as shown in Fig. 1. We assume

a synchronous slotted system, in which each source $i=$ 1,2 buffers the incoming packets in queue $Q_{i}$ with backlog $q_{i}(t)$ at time (slot) $t$. Each source $i$ chooses the service rate $\mu_{i}(t)$ at time $t$ (namely, the number of packets/bits or more generally data units injected to the system) such that

$$
\left(\mu_{1}(t), \mu_{2}(t)\right) \in C(t),
$$

where $C(t)$ is the rate region achievable by network coding at time $t$. In this paper, we focus on a simple rate region $C(t)=\left\{\left(\mu_{1}(t), \mu_{2}(t)\right): 0 \leq \mu_{i}(t) \leq \mu_{i}^{\max }(t), i=1,2\right\}$ achievable over the relay $R$. This represents the case of orthogonal channels from sources to the relay. The extension to more general rate regions can further couple the service rates in addition to the coupling effect of network coding, which poses the main interest in this paper. The queue length at source $i=1,2$ evolves as

$$
q_{i}(t+1)=\max \left(q_{i}(t)-\mu_{i}(t), 0\right)+a_{i}(t),
$$

where $a_{i}(t)$ is the number of bits/packets arriving at source queue $Q_{i}$ at time $t$. For each source $i$, we assume that $a_{i}(t)$ is generated via an ergodic process and let $\lambda_{i}$ denote its long-term average.

Alternatively, the queue dynamics can be written as

$$
q_{i}(t+1)=q_{i}(t)-\tilde{\mu}_{i}(t)+a_{i}(t)
$$

where the actual transmission rate of source $i$ is

$$
\tilde{\mu}_{i}(t)=\min \left(\mu_{i}(t), q_{i}(t)\right), \quad i=1,2,
$$

since the number of transmitted packets is limited by the number of packets available in the queue. Note that the rate $\mu_{i}(t), i=1,2$, is sufficient to optimize the throughput rates, since sources can simply perform zero padding in the absence of packets. However, we are interested in minimizing the cost, which is a function of the number of transmitted packets. Therefore, we use the actual transmission rate, namely $\tilde{\mu}_{i}(t)$, that is bounded by the number packets available in the source queues. The instantaneous rate region is defined as

$$
\begin{array}{r}
\tilde{C}(t)=\left\{\tilde{\mu}_{1}(t), \tilde{\mu}_{2}(t):\left(\tilde{\mu}_{1}(t), \tilde{\mu}_{2}(t)\right) \in C(t),\right. \\
\left.\tilde{\mu}_{i}(t) \leq q_{i}(t), i=1,2\right\} .
\end{array}
$$

We assume that relay $R$ does not buffer the incoming packets in queues and immediately forwards any received data over a single channel which is orthogonal to the channels used by each source. Specifically, the relay will use network coding to transmit an equal number of packets per time-slot from both sources simultaneously at common rate $\min _{j=1,2}\left(\tilde{\mu}_{j}(t)\right)$. Any residual traffic is then routed in uncoded form only from one source $i$ with rate $\tilde{\mu}_{i}(t)-$ $\min _{j=1,2}\left(\tilde{\mu}_{j}(t)\right)$. Note that the network coding operation can be carried out at packet or signal levels, i.e., relay $R$ either decodes, network-codes and transmits the incoming packets, or simply amplifies-and-forwards the received signals as in analog network coding [3]. Decoding is accomplished by combining the received network-coded packets with that source's individual packets. We do not consider the network coding overhead to notify sources whether the transmitted packet is network-coded or in plain form, and assume that this overhead is negligible compared to packet size.

Each use of the relay $R$ is assumed to incur a cost, which could, for example, represent the energy expended by the relay. We assume that the relay cost is partitioned among the two sources depending on the rate allocation such that the cost of source $i=1,2$ for using the relay $R$ with rate $\tilde{\mu}_{i}$ is

$$
\begin{aligned}
J_{i}\left(\tilde{\mu}_{1}(t), \tilde{\mu}_{2}(t)\right)= & c_{i}(t)\left(\min _{j=1,2}\left(\tilde{\mu}_{j}(t)\right)\right) \\
& +d_{i}(t)\left(\tilde{\mu}_{i}(t)-\min _{j=1,2}\left(\tilde{\mu}_{j}(t)\right)\right),
\end{aligned}
$$

where $c_{i}(t)$ and $d_{i}(t)$ are the costs charged by the relay $R$ to deliver one packet by network coding and routing, respectively. For most of the following we focus on the case where $c_{i}(t)=\frac{1}{2}$, and $d_{i}(t)=1$. This models the case where the cost per unit time of operating the relay is the same for both routing and network coding and in the network coding case the cost is equally shared by both sources, whereas any source is solely responsible for the cost of packets routed by the relay. Then, the individual cost (6) can be rewritten as

$$
J_{i}\left(\tilde{\mu}_{1}(t), \tilde{\mu}_{2}(t)\right)=\tilde{\mu}_{i}(t)-\frac{1}{2} \min _{j=1,2}\left(\tilde{\mu}_{j}(t)\right)
$$

such that the total cost is simplified to

$$
\sum_{i=1}^{2} J_{i}\left(\tilde{\mu}_{1}(t), \tilde{\mu}_{2}(t)\right)=\max _{i=1,2}\left(\tilde{\mu}_{i}(t)\right) .
$$

\section{Centralized Cost Optimization}

We start with the case where a centralized controller has access to all system parameters along with the queue backlog information, and makes the transmission decisions for both sources. The main objective is to minimize the total cost for both sources while ensuring that the average delay is no greater than a given value $D$. This leads to the following optimization problem:

Total Minimum Cost Problem:

$$
\begin{array}{r}
\min _{\left(\tilde{\mu}_{1}(t), \tilde{\mu}_{2}(t)\right) \in \tilde{C}(t), t \geq 0} \lim _{t \rightarrow \infty} \frac{1}{t} \sum_{\tau=0}^{t-1} \sum_{i=1}^{2} \mathbb{E}\left[J_{i}\left(\tilde{\mu}_{1}(\tau), \tilde{\mu}_{2}(\tau)\right)\right] \\
\text { s.t. } \lim _{t \rightarrow \infty} \frac{1}{t} \sum_{\tau=0}^{t-1} \sum_{i=1}^{2} \frac{\mathbb{E}\left[q_{i}(\tau)\right]}{\lambda_{1}+\lambda_{2}}<D .
\end{array}
$$

Here, the average delay constraint follows from the ratio of the average queue length to the total arrival rate according to 
Little's theorem [19]. Note also that the objective in (PC) is equivalent to the average cost per packet by normalizing by the total long-term arrival rate $\lambda_{1}+\lambda_{2}$. Let $\mathcal{P}^{*}(D)$ denote the solution to (PC) as a function of the delay constraint $D$, i.e. the cost-delay trade-off. In general this will be a decreasing function of $D$ and as $D \rightarrow \infty$, it will yield the minimum cost solution subject to the queue's being stable.

In principle, for a given delay constraint, Problem (PC) can be solved via dynamic programming. However, such a solution quickly becomes intractable except for very simple arrival processes and furthermore, requires a priori knowledge of arrival statistics. Instead, we will follow the approach in [10] and use Lyapunov stability arguments to yield an approximate solution to (PC) with provable approximation bounds. This approach is based on generalizing the classical back-pressure algorithm from [8] which is guaranteed to stabilize the packet queues if this is possible. This throughputoptimal solution is given by

$$
\max _{\left(\mu_{1}(t), \mu_{2}(t)\right) \in C(t), t \geq 0} \sum_{i=1}^{2} q_{i}(t) \mu_{i}(t),
$$

which can be rewritten as

$$
\max _{\left(\tilde{\mu}_{1}(t), \tilde{\mu}_{2}(t)\right) \in \tilde{C}(t), t \geq 0} \sum_{i=1}^{2} q_{i}(t) \tilde{\mu}_{i}(t) .
$$

Note that (9)-(10) are not optimal with respect to minimizing the time-average cost in (PC) as shown in the following example:

Example 1: Let $a_{1}(t)=1, a_{2}(t)=2$ for even $t$, and $a_{1}(t)=2, a_{2}(t)=1$ for odd $t$. For $c_{i}(t)=\frac{1}{2}$ and $d_{i}(t)=$ 1 , the back-pressure solution is $\mu_{1}(t)=1, \mu_{2}(t)=2$ for even $t$, and $\mu_{1}(t)=2, \mu_{2}(t)=1$ for odd $t$. The longterm average costs per packet $J_{1}$ and $J_{2}$ are $\frac{2}{3}$. However, if sources accumulate packets over time slots and transmit such that relay $R$ performs network coding only, the decisions $\mu_{1}(t)=1, \mu_{2}(t)=1$ for even $t$ and $\mu_{1}(t)=2, \mu_{2}(t)=2$ for odd $t$ make the long-term average costs approach the lower bound $\frac{1}{2}$ (which is achieved, if relay $R$ only performs network coding such that the relay cost for each networkcoded packet is always equally shared by both sources).

The main idea in [10] is to augment the policy in (10) with an additional term to reflect the cost. The resulting problem is given by

$$
\begin{aligned}
\max _{\left(\tilde{\mu}_{1}(t), \tilde{\mu}_{2}(t)\right) \in \tilde{C}(t)} & \left(q_{1}(t) \tilde{\mu}_{1}(t)+q_{2}(t) \tilde{\mu}_{2}(t)\right. \\
& \left.-V \max \left(\tilde{\mu}_{1}(t), \tilde{\mu}_{2}(t)\right)\right),
\end{aligned}
$$

where $V$ is a control parameter to tune the trade-off between the average queue backlog and the distance from the minimum achievable cost. In (P1), maximizing the first term $q_{1}(t) \tilde{\mu}_{1}(t)+q_{2}(t) \tilde{\mu}_{2}(t)$ is due to backpressure algorithm in order to stabilize the source queues, whereas minimizing the second term $\max \left(\tilde{\mu}_{1}(t), \tilde{\mu}_{2}(t)\right)$ serves the purpose of moving the stable solutions to the minimum achievable cost.

Theorem 1: At any time slot $t$, the solution to (P1) is given by the algorithm (A1) as follows:

$$
\begin{aligned}
& \left(\tilde{\mu}_{i}(t), \tilde{\mu}_{j}(t)\right)= \\
& \begin{cases}(0,0), & \text { if } V>q_{1}(t)+q_{2}(t), \\
\left(u_{c}(t), u_{c}(t)\right), & \text { if } q_{1}(t)+q_{2}(t)>V \geq \max \left(q_{1}(t), q_{2}(t)\right), \\
\left(u_{i}(t), u_{c}(t)\right), & \text { if } q_{i}(t)>V \geq q_{j}(t), j \neq i, \\
\left(u_{i}(t), u_{c}(t)\right), & \text { if } V \leq \min \left(q_{1}(t), q_{2}(t)\right), \\
& \left(q_{i}(t)-V\right) u_{i}(t)+q_{j}(t) u_{c}(t) \geq \\
& \left(q_{j}(t)-V\right) u_{j}(t)+q_{i}(t) u_{c}(t), j \neq i,\end{cases}
\end{aligned}
$$

where

$$
\begin{aligned}
& u_{i}(t)=\min \left(q_{i}(t), \mu_{i}^{\max }(t)\right), \\
& u_{c}(t)=\min \left(u_{1}(t), u_{2}(t)\right) .
\end{aligned}
$$

Proof: The objective function to be maximized depends on which source is assigned a higher rate, and it is either equal to $\left(q_{1}(t)-V\right) \tilde{\mu}_{1}(t)+q_{2}(t) \tilde{\mu}_{2}(t)$, if $\tilde{\mu}_{1}(t) \geq \tilde{\mu}_{2}(t)$ or $\left(q_{2}(t)-V\right) \tilde{\mu}_{2}(t)+q_{1}(t) \tilde{\mu}_{1}(t)$, if $\tilde{\mu}_{2}(t) \geq \tilde{\mu}_{1}(t)$. Consider the case with $q_{i}(t)>V$ and $q_{j}(t)<V$. For $\tilde{\mu}_{i}(t) \geq \tilde{\mu}_{j}(t)$, the objective function is greater than or equal to $\left(q_{1}(t)+\right.$ $\left.q_{2}(t)-V\right) u_{c}(t)$, whereas the objective function is at most $\left(q_{1}(t)+q_{2}(t)-V\right) u_{c}(t)$ for $\tilde{\mu}_{j}(t) \geq \tilde{\mu}_{i}(t)$. Accordingly, the rate allocation with $\mu_{i}(t) \geq \mu_{j}(t)$ is selected with $\tilde{\mu}_{i}(t)$ equal to $u_{i}(t)$ and $\tilde{\mu}_{j}(t)$ equal to $u_{c}(t)$.

Without loss of generality, consider the case with $\tilde{\mu}_{i}(t) \geq$ $\tilde{\mu}_{j}(t)$. Then, $\max \left(\tilde{\mu}_{1}(t), \tilde{\mu}_{2}(t)\right)=\tilde{\mu}_{i}(t)$, and the objective function is $\left(q_{i}(t)-V\right) \tilde{\mu}_{i}(t)+q_{j}(t) \tilde{\mu}_{j}(t)$.

If $q_{i}(t)<V$, the optimal solution should minimize $u_{i}(t)$, since the multiplier associated with the rate of source $i$ is negative. However, the special case with $\tilde{\mu}_{i}(t)=\tilde{\mu}_{j}(t)$ should be also taken into account. If $q_{j}(t)>\left|q_{i}(t)-V\right|$, or equivalently if $q_{1}(t)+q_{2}(t)>V$, then the optimal rate allocation is $\tilde{\mu}_{i}(t)=\tilde{\mu}_{j}(t)=u_{c}(t)$. On the other hand, if $q_{j}(t)<\left|q_{i}(t)-V\right|$, i.e., if $q_{1}(t)+q_{2}(t)<V$, the optimal rate allocation is $\tilde{\mu}_{i}(t)=\tilde{\mu}_{j}(t)=0$.

Finally, if both $q_{i}(t)$ and $q_{j}(t)$ exceed $V$, rates should be selected from one of the two possibilities in the fourth case of (A1) to maximize the overall objective function.

The cost-delay trade-offs for Algorithm (A1) are evaluated in Theorem 2:

Theorem 2: For Algorithm (A1), the average queue back$\log$ and the average packet delay are proportional to $V$, while the distance of the average cost from the minimum attainable cost is proportional to $\frac{1}{V}$ :

$$
\begin{aligned}
& \lim _{M \rightarrow \infty} \frac{1}{M} \sum_{\tau=0}^{M-1} \sum_{i=1}^{2} J_{i}\left(\tilde{\mu}_{1}(\tau), \tilde{\mu}_{2}(\tau)\right) \leq J^{*}+\frac{B_{1}}{V}, \\
& \lim _{M \rightarrow \infty} \frac{1}{M} \sum_{\tau=0}^{M-1} \sum_{i=1}^{2} \mathbb{E}\left[q_{i}(\tau)\right] \leq \frac{B_{2}+\frac{V}{2}}{\epsilon}
\end{aligned}
$$

where $J^{*}$ is the optimal cost per time slot, $B_{1}$ and $B_{2}$ are positive constants, and $\lambda_{i}$ satisfies $\left(\lambda_{i}+\epsilon\right) \in C(t), i=1,2$, at any time $t$ for an arbitrarily small positive constant $\epsilon$.

Proof: (Sketch) We provide only a general sketch of the proof for brevity. The proof follows from the Lyapunov drift analysis with performance optimization arguments, as 
in [10]. First, the Lyapunov drift (namely, the time-average difference of Lyapunov functions for queue backlogs) is expressed for queue dynamics, and the average cost is added to the resulting drift formulation. This leads to Lyapunov drift conditions for stability. Next, we utilize the properties of the rate allocation algorithm to show that these conditions are satisfied, leading to bounds on the average queue lengths and consequently on the average values of delay and cost.

The optimal cost per packet $J_{p}^{o p t}=\frac{J^{*}}{\lambda_{1}+\lambda_{2}}$ is given by $\frac{\max \left(\lambda_{1}, \lambda_{2}\right)}{\lambda_{1}+\lambda_{2}}$, and it is reduced to $\frac{1}{2}$ for $\lambda_{1}=\lambda_{2}$.

For $q_{1}(t)+q_{2}(t)>V>\max \left(q_{1}(t), q_{2}(t)\right)$, relay $R$ performs only network coding and does not forward any uncoded packet, which results in the energy-optimal operation. As $V$ increases, sources tend to transmit less. On the other hand, as $V$ decreases, relay $R$ also forwards the residual traffic in uncoded form, which would result in the throughput-optimal operation of the classical back-pressure algorithm.

\section{Individual Cost Optimization}

Next we consider a scenario in which the individual nodes decide on their own rate allocations given full knowledge of the other user's queue size. Each user $i=1,2$ would like to solve the following individual optimization problem:

Individual Minimum Cost Problem:

$\min _{\tilde{\mu}_{i}(t):\left(\tilde{\mu}_{1}(t), \tilde{\mu}_{2}(t)\right) \in \tilde{C}(t), t \geq 0} \lim _{t \rightarrow \infty} \frac{1}{t} \sum_{\tau=0}^{t-1} \mathbb{E}\left[J_{i}\left(\left(\tilde{\mu}_{1}(\tau), \tilde{\mu}_{2}(\tau)\right)\right]\right.$

(PCI)

$$
\text { s.t. } \lim _{t \rightarrow \infty} \frac{1}{t} \sum_{\tau=0}^{t-1} \frac{\mathbb{E}\left[q_{i}(\tau)\right]}{\lambda_{i}}<D_{i} .
$$

In this setting, the two users can be viewed as paying a non-cooperative game, in which (PCI) reflects the pay-off of user $i$. In principle, this can be viewed as a stochastic game which can again be solved using dynamic programming ideas. However, again such an approach quickly becomes intractable and we instead consider a Lyapunov drift formulation as as we did in (P1) for the centralized optimization problem. Furthermore, we assume that in each time-slot, the sources play a single-stage game against each other with the corresponding pay-off function. ${ }^{1}$ In other words, given $\tilde{\mu}_{j}$, $j \neq i$, source $i=1,2$ chooses the individual strategy $\tilde{\mu}_{i}(t)$ to solve:

$\max _{\tilde{\mu}_{i}(t):\left(\tilde{\mu}_{1}(t), \tilde{\mu}_{2}(t)\right) \in \tilde{C}(t), t \geq 0}\left(q_{i}(t) \tilde{\mu}_{i}(t)-V_{i} J_{i}\left(\tilde{\mu}_{1}(t), \tilde{\mu}_{2}(t)\right)\right)$.

Minimizing the first term $q_{i}(t) \tilde{\mu}_{i}(t)$ in the individual objective function is to stabilize the queue of source $i$, whereas minimizing the second term $V_{i} J_{i}\left(\tilde{\mu}_{1}(t), \tilde{\mu}_{2}(t)\right)$ moves the stable solution to the optimal cost value. Define $\tilde{J}_{i}\left(\tilde{\mu}_{1}(t), \tilde{\mu}_{2}(t)\right)=q_{i}(t) \tilde{\mu}_{i}(t)-V_{i} J_{i}\left(\tilde{\mu}_{1}(t), \tilde{\mu}_{2}(t)\right)$.

\footnotetext{
${ }^{1}$ One advantage of this formulation is that it does not require any knowledge of the long-term arrival statistics of either source.
}

Definition 1: A pair of strategies $\left\{\tilde{\mu}_{i}^{*}\right\}$ are a Nash equilibrium for the resulting stage game if, for each player $i$,

$$
\tilde{J}_{i}\left(\tilde{\mu}_{i}^{*}, \tilde{\mu}_{-i}^{*}\right) \geq \tilde{J}_{i}\left(\tilde{\mu}_{i}, \tilde{\mu}_{-i}^{*}\right), \text { for all } \tilde{\mu}_{i} \in \tilde{C}(t),
$$

where " $-i$ " denotes the player other than player $i$.

For any source $i=1,2$, the strategy space is a non-empty convex subset of an Euclidean space and the utility function is continuous in $\left(\tilde{\mu}_{1}, \tilde{\mu}_{2}\right)$ and quasi-concave in $\tilde{\mu}_{i}$. Therefore, there exists pure strategy Nash equilibrium at any time slot $t$ for given $q_{i}(t), i=1,2$.

Theorem 3: The Nash equilibrium strategy (A2) of any source $i=1,2$ as solution to $(\mathbf{P} 2)$ is given by:

$$
\begin{aligned}
& \tilde{\mu}_{i}(t)= \\
& \begin{cases}0, & \text { if } q_{i}(t)<\frac{V_{i}}{2}, \\
u_{i}(t), & \text { if } q_{i}(t) \geq V_{i}, \\
0, & \text { if } \frac{V_{i}}{2} \leq q_{i}(t)<V_{i}, q_{j}(t)<\frac{V_{j}}{2}, j \neq i, \\
u_{c}(t), & \text { if } \frac{V_{i}}{2} \leq q_{i}(t)<V_{i}, q_{j}(t) \geq V_{j}, j \neq i, \\
0 \text { or } u_{c}(t), & \text { if } \frac{V_{i}}{2} \leq q_{i}(t)<V_{i}, i=1,2 .\end{cases} \\
& \quad \text { Proof: Note that } J_{i}\left(\tilde{\mu}_{1}(t), \tilde{\mu}_{2}(t)\right)=\tilde{\mu}_{i}(t)- \\
& \frac{1}{2} \min _{j=1,2}\left(\tilde{\mu}_{j}(t)\right) \text { is equal to } \tilde{\mu}_{i}(t)-\frac{1}{2} \tilde{\mu}_{j}(t), \text { if } \tilde{\mu}_{i}(t) \geq \\
& \tilde{\mu}_{j}(t), \text { or equal to } \frac{1}{2} \tilde{\mu}_{i}(t), \text { if } \tilde{\mu}_{j}(t) \geq \tilde{\mu}_{i}(t) .
\end{aligned}
$$

Without loss of generality, consider the former case, in which the objective function is equal to $\left(q_{i}(t)-V_{i}\right) \tilde{\mu}_{i}(t)+$ $\frac{V_{i}}{2} q_{j}(t) \tilde{\mu}_{j}(t)$ for source $i$, and equal to $\left(q_{j}(t)-\frac{V_{j}}{2}\right) \tilde{\mu}_{j}(t)$ for source $j \neq i$. The latter case follows directly by interchanging $i$ and $j$. If $q_{i}(t)>V_{i}, i=1,2$, the maximum possible value for $\tilde{\mu}_{i}(t)$ is selected as $u_{i}(t)$, since the multiplier associated with the rate of source $i$ will be always positive. Similarly, if $q_{i}(t)<\frac{V_{i}}{2}, i=1,2$, the minimum possible value for $\tilde{\mu}_{i}(t)$ is selected as 0 , since the multiplier associated with the rate of source $i$ will be always negative.

Consider the case with $\frac{V_{i}}{2}<q_{i}(t)<V_{i}$. If $q_{j}(t)<\frac{V_{j}}{2}$, source $j$ will not transmit and the best strategy of source $i$ is to reduce $u_{i}(t)$ to 0 . On the other hand, if $q_{j}(t)>V_{j}$, the best strategy of source $j$ is to transmit. Then, source $i$ either tends to decrease its rate to $u_{c}(t)$ for $\tilde{\mu}_{i}(t) \geq \tilde{\mu}_{j}(t)$, or tends to increase its rate to $u_{c}(t)$ for $\tilde{\mu}_{j}(t) \geq \tilde{\mu}_{i}(t)$. In effect, source $i$ selects the rate $u_{c}(t)$ for either case.

For $\frac{V_{i}}{2}<q_{i}(t)<V_{i}, i=1,2$, there are multiple Nash equilibria, since the equilibrium can also be established at $\mu_{i}(t)=0, i=1,2$, in addition to $\mu_{i}(t)=u_{c}(t), i=1,2$.

\section{Dynamic Pricing at the Relay}

In this section, we consider the case where sources have no information about each other's queue backlog in contrast to (P2). For that purpose, any source $i=1,2$ assumes that the opponent source $j \neq i$ plays a fixed strategy. The worst-case response of the opponent $j$ is $\tilde{\mu}_{j}=0$ such that there is no possibility of performing network coding to reduce the relay cost. If source $i=1,2$ assumes that source $j \neq i$ plays the worst-case strategy, it ends up with the optimization problem:

$$
\max _{\tilde{\mu}_{i}(t):\left(\tilde{\mu}_{i}(t), 0\right) \in \tilde{C}(t), t \geq 0}\left(q_{i}(t) \tilde{\mu}_{i}(t)-V_{i} J_{i}\left(\tilde{\mu}_{i}(t), 0\right)\right) .
$$


The solution to (P3) is given by:

$$
\tilde{\mu}_{i}(t)= \begin{cases}0, & \text { if } q_{i}(t)<V_{i}, \\ u_{i}(t), & \text { if } q_{i}(t) \geq V_{i} .\end{cases}
$$

(A3) simply involves comparing the individual queue backlog with a threshold that is independent of the opponent's queue backlog or transmission decision. A similar algorithm (A4) can be defined by assuming that the opponent plays the best strategy. In (P2), the best-case response of the opponent source $j$ is $\tilde{\mu}_{j}=\mu_{j}^{\max }(t)$ such that for packets of source $i$ there is always an opportunity to perform network coding such as to reduce the relay cost. The resulting problem is

$\max _{\tilde{\mu}_{i}(t):\left(\tilde{\mu}_{i}(t), \mu_{j}^{\max }(t)\right) \in C(t), j \neq i}\left(q_{i}(t) \tilde{\mu}_{i}(t)-V_{i} J_{i}\left(\tilde{\mu}_{i}(t), \mu_{j}^{\max }(t)\right)\right)$.

The solution (A4) to (P4) is given by:

$$
\begin{aligned}
& \tilde{\mu}_{i}(t)= \\
& \begin{cases}0, & \text { if } q_{i}(t)<\frac{V_{i}}{2}, \\
\mu_{j}^{\max }(t), & \text { if } u_{i}(t) \geq \mu_{j}^{\max }(t), \frac{V_{i}}{2} \leq q_{i}(t)<V_{i}, j \neq i, \\
u_{i}(t), & \text { if } u_{i}(t) \geq \mu_{j}^{\max }(t), q_{i}(t) \geq V_{i}, j \neq i, \\
u_{i}(t), & \text { if } u_{i}(t)<\mu_{j}^{\max }(t), q_{i}(t) \geq \frac{V_{i}}{2}, j \neq i .\end{cases}
\end{aligned}
$$

So far, we assumed fixed weights $c_{i}(t)=\frac{1}{2}$ for network coding and $d_{i}(t)=1$ for routing. Instead, relay $R$ can apply dynamic pricing by selecting the weights $c_{i}(t)$ and $d_{i}(t)$ in (6) as functions of queue backlogs $q_{1}(t)$ and $q_{2}(t)$ to adapt the distributed energy costs to the centralized optimal solutions. For that purpose, relay penalizes the difference between the data rates transmitted from each source thereby increasing the network coding opportunities and minimizing the relay cost. The individual cost for node $i$ is changed to

$$
\begin{gathered}
J_{i}\left(\tilde{\mu}_{1}(t), \tilde{\mu}_{2}(t)\right)=c_{i}(t) \min _{j=1,2} \tilde{\mu}_{j}(t)+d_{i}(t)\left(\tilde{\mu}_{i}(t)\right. \\
\left.-\min _{j=1,2} \tilde{\mu}_{j}(t)\right)+e_{i}(t)\left(\max _{j=1,2} \tilde{\mu}_{j}(t)-\min _{j=1,2} \tilde{\mu}_{j}(t)\right),
\end{gathered}
$$

where $e_{i}(t)$ is the weight to equalize the transmission rates for both sources. The individual optimization problem is

$$
\begin{aligned}
& \max _{\tilde{\mu}_{i}(t):\left(\tilde{\mu}_{i}(t)\right) \in C(t), t \geq 0}\left(q_{i}(t) \tilde{\mu}_{i}(t)-d_{i}(t) \tilde{\mu}_{i}(t)\right. \\
& \left.+\left(d_{i}(t)+e_{i}(t)-c_{i}(t)\right) \min _{j=1,2} \tilde{\mu}_{j}(t)-e_{i}(t) \max _{j=1,2} \tilde{\mu}_{j}(t)\right),
\end{aligned}
$$

where the rate weights $c_{i}(t), d_{i}(t)$ and $e_{i}(t)$ are adjusted by the relay $R$ depending on the queue backlogs $q_{1}(t)$ and $q_{2}(t)$, and the sources play the worst-case response without knowing each other's queue backlogs.

Theorem 4: Given the queue backlogs $q_{i}(t), i=1,2$, and threshold parameter $V$, the rate weights $c_{i}(t), d_{i}(t)$ and $e_{i}(t)$ for (A3) to achieve the optimal solutions (A1) satisfy:

$$
\begin{aligned}
& \text { If } V>q_{1}(t)+q_{2}(t): \\
& \quad q_{i}(t)<c_{i}(t)<d_{i}(t)+e_{i}(t), i=1,2
\end{aligned}
$$

$$
\begin{aligned}
& \text { If } q_{1}(t)+q_{2}(t) \geq V>\max \left(q_{1}(t), q_{2}(t)\right): \\
& d_{i}(t)+e_{i}(t)>q_{i}(t), c_{i}(t)>d_{i}(t)+e_{i}(t), i=1,2 \\
& \text { If } q_{i}(t) \geq V>q_{j}(t), j \neq i: \\
& d_{i}(t)+e_{i}(t)<q_{i}(t), d_{j}(t)+e_{j}(t)>q_{j}(t), \\
& c_{j}(t)>d_{j}(t)+e_{j}(t),
\end{aligned}
$$

Proof: The idea is to arrange the coefficients in (16) and adjust the weights $c_{i}(t), d_{i}(t)$ and $e_{i}(t)$ to mimic the behavior of the centralized case (A1). The objective function to be maximized depends on which source is assigned a higher rate, and is equal to $\left(q_{i}(t)-d_{i}(t)-e_{i}(t)\right) \tilde{\mu}_{i}(t)+$ $\left(d_{i}(t)+e_{i}(t)-c_{i}(t)\right) \tilde{\mu}_{j}(t)$, if $\tilde{\mu}_{i}(t) \geq \tilde{\mu}_{j}(t)$, or $\left(q_{i}(t)+\right.$ $\left.e_{i}(t)-c_{i}(t)\right) \tilde{\mu}_{i}(t)-e_{i}(t) \tilde{\mu}_{j}(t)$, if $\tilde{\mu}_{i}(t) \leq \tilde{\mu}_{j}(t)$.

For brevity, we will consider two subcases. First, assume $V>q_{1}(t)+q_{2}(t)$, where the solution should be $\tilde{\mu}_{j}(t)=$ $\tilde{\mu}_{i}(t)=0$ from (A1). If $c_{i}(t)<d_{i}(t)+e_{i}(t)$, assuming $\tilde{\mu}_{i}(t) \geq \tilde{\mu}_{j}(t)$, the the worst-case response is $\tilde{\mu}_{j}(t)=0$, and if $d_{i}(t)+e_{i}(t)>q_{i}(t), \tilde{\mu}_{i}(t)$ is selected as 0 , resulting in an objective value of 0 . Next, consider the assumption $\tilde{\mu}_{i}(t) \leq$ $\tilde{\mu}_{j}(t)$, where the worst case response is $\tilde{\mu}_{j}(t)=0$ for $e_{i}<0$ and $\tilde{\mu}_{j}(t)=u_{j}(t)$ if $e_{i}>0$. For $e_{i}<0$, we have the desired solution $\tilde{\mu}_{i}(t)=0$ from the initial assumption $\tilde{\mu}_{i}(t) \leq \tilde{\mu}_{j}(t)$. For $e_{i}>0$, the maximum value of the objective function is $\left(q_{i}(t)+e_{i}(t)-c_{i}(t)\right) u_{c}(t)-e_{i}(t) u_{c}(t)=\left(q_{i}(t)-c_{i}(t)\right) u_{c}(t)$. With $q_{i}(t)<c_{i}(t)$, note that the resulting objective value is negative and the overall rate selection is $\tilde{\mu}_{i}(t)=0$. Changing the indices $i$ and $j$ results in the symmetric operation, and the centralized solution is achieved for this case.

Next, assume $q_{1}(t)+q_{2}(t)>V>\max \left(q_{1}(t), q_{2}(t)\right)$. For $\tilde{\mu}_{i}(t) \geq \tilde{\mu}_{j}(t)$, by selecting $c_{i}(t)>d_{i}(t)+e_{i}(t)$, the worst-case response is to maximize $\tilde{\mu}_{j}(t)$. However, if $d_{i}(t)+e_{i}(t)>q_{i}(t)$, source $i$ tends to reduce $\tilde{\mu}_{i}(t)$ to $u_{c}(t)$ because of the worst-case response from source $j$. The resulting objective value is equal to $\left(q_{i}(t)-c_{i}(t)\right) u_{c}(t)$. It can be shown that the maximum objective value achieved by assuming $\tilde{\mu}_{i}(t) \leq \tilde{\mu}_{j}(t)$ is also equal to $\left(q_{i}(t)-c_{i}(t)\right) u_{c}(t)$, with $\tilde{\mu}_{i}(t)=u_{c}(t)$. Hence, the overall solution is $\tilde{\mu}_{i}(t)=$ $u_{c}(t)$. The other two subcases for (A1) can be derived similarly. By setting $d_{i}(t)+e_{i}(t)<q_{i}(t)$, the rates of source $i$ are increased in accordance with the solution of (A1).

\section{Cost Sharing With 1-Bit Queue Information}

For the individual cost optimization problem, we can further simplify the transmission decisions by limiting the necessary queue information to one bit, which distinguishes whether the opponent's queue exceeds a threshold, or not. For that purpose, we present the following algorithm (A5):

Let $\mu^{\max }=\min _{j=1,2} \mu_{j}^{\max }$. The system starts with threshold equal to $V_{i}=\mu^{\max }+\mu_{i}^{\max }$ for each source $i$. 
Then, source $i=1,2$ decides to transmit, if its individual backlog satisfies $q_{i}(t) \geq V_{i}$. When the relay observes that both sources have transmitted at least once, then it knows that $q_{i}(t) \geq \mu^{\max }$ for both $i=1,2$, so it reduces the threshold to the common maximum service rate $\mu^{\text {max }}$ knowing that both sources will operate with network coding. Afterwards, the thresholds are set to $V_{i}$ again, and the operation continues.

The relay cost can be further improved as follows. Each source $i$ starts with one threshold $V_{i}$ (which is greater than $\left.\mu_{i}^{\max }\right)$, and transmits with rate $\mu_{i}^{\max }$ if its queue size exceeds $V_{i}$. Source $i$ only needs to know whether the queue backlog $q_{j}(t)$ of the other source $j \neq i$ exceeds the minimum of the maximum service rates of both users $\mu^{\max }=\min _{j=1,2} \mu_{j}^{\max }$, or not. If so, a second threshold $\mu_{\max }$ is introduced. Then, if the queue size of source $i$ is between the two thresholds $\mu_{\max }$ and $V_{i}$, source $i$ transmits with rate $\mu_{\max }$. The resulting algorithm (A6) is given by:

$$
\tilde{\mu}_{i}(t)= \begin{cases}0, & \text { if } q_{i}(t)<\mu^{\max }, \\ 0, & \text { if } \mu^{\max } \leq q_{i}(t)<V_{i}, \\ & q_{j}(t)<\mu^{\max }, j \neq i, \\ \mu^{\max ,}, & \text { if } \mu^{\max } \leq q_{i}(t)<V_{i}, \\ & q_{j}(t)>\mu^{\max }, j \neq i, \\ \mu_{i}^{\max }, & \text { if } q_{i}(t) \geq V_{i} .\end{cases}
$$

We will show in Section VIII that the cost performance of (A6) is very close to the centralized algorithm, especially as we increase the thresholds $V_{i}, i=1,2$.

(A6) has a similar structure as the algorithm proposed in [7], where two queues in the relay store packets incoming from two sources. If both relay queues are nonempty, two packets, one from each relay queue, are network-coded and transmitted by the relay. If one of the relay queues is empty, there is no transmission unless the backlogged queue length exceeds a fixed threshold (the buffer capacity). However, in [7], the system is not slotted and the scheduling decisions are carried out at the instants of packet arrivals. Between two consecutive scheduling instances, at most one packet can arrive at one of the two sources, and the combined queue state can change at most by 1 . This particular model allows a Markov chain analysis for the decoupled source queues. However, in this paper we assume that the number of packet arrivals per time slot may be arbitrary, and this leads to more complex transitions between the two queue states.

\section{COST Sharing WITH LOCAL QUEUE INFORMATION}

Next, we assume that sources do not have any information about each other's queue backlogs. First, we focus on the class of algorithms (A7), where any source $i$ does not transmit, until the individual queue backlogs exceed a threshold $T$, and transmits with probability $p_{i}$, if the individual queue backlog is greater than $T$. The resulting algorithm (A7) is

$$
\tilde{\mu}_{i}(t)= \begin{cases}0, & \text { if } q_{i}(t)<T, \\ u_{i}(t) \text { with prob. } p_{i}, \text { or } & \\ 0 \text { with prob. }\left(1-p_{i}\right) & \text { if } q_{i}(t) \geq T,\end{cases}
$$

We assume that the maximum service rates of both sources are identical to $\mu^{\max }$. One restriction on $p_{i}$ is that it should be large enough to ensure that the system operates under negative drift after $q_{i}(t)$ exceeds $\max \left(\mu^{\max }, T\right)$, i.e., we need $p_{i}>\frac{\lambda_{i}}{\mu^{\max }}$. If $T \geq \mu^{\max }$, the queueing system can be modeled with two states: states with queue backlogs less than $\mu^{\max }$ and states with queue backlogs greater than $\mu^{\max }$. This leads to a bulk queue system model, where the service rate is $p_{i} \mu^{\max }$ and the arrival rate is $\lambda_{i}$. If we denote $q_{i}$ as the stationary queue backlog of source $i=1,2$, then $P\left(q_{i}<\mu^{\max }\right)=1-\frac{\lambda_{i}}{p_{i} \mu^{\max }}$ from the Little's theorem [19]. If a source transmits a packet, the average cost depends on the probability that the other source also transmits. Then, for each source $i$ the average cost per packet is

$$
\begin{aligned}
E\left[J_{i}\right] & =0.5 \times\left(\frac{\lambda_{i}}{p_{i} \mu^{\max }}\right) p_{i}+1 \times\left(1-\frac{\lambda_{i}}{p_{i} \mu^{\max }}\right) p_{i} \\
& =1-0.5 \frac{\lambda_{i}}{\mu^{\max }} .
\end{aligned}
$$

where $J_{i}$ denotes cost per packet for source $i$.

When the queue backlog is between 1 and $\mu^{\max }$, we can allow the sources to transmit with some non-zero probability (either fixed or queue state dependent). In this algorithm (A8), the transmission decisions are given by:

$$
\tilde{\mu}_{i}(t)= \begin{cases}0, & \text { if } q_{i}(t)=0 \\ u_{i}(t) \text { with prob. } p_{i}, \text { or } & \\ 0 \text { with prob. }\left(1-p_{i}\right) & \text { if } q_{i}(t) \geq 1,\end{cases}
$$

In (A8), given that a source transmits, the probability that the other source transmits is higher compared to (A7). On the other hand, when sources transmit and their queue backlogs are less than $\mu_{\max }$, there might be a mismatch between the number of packets transmitted from each source, and the cost per packet might be greater than the optimal value $\frac{1}{2}$ even if both sources transmit simultaneously.

\section{COMPARISON OF COST-DELAY TRADE-OFFS}

We compare the cost and delay performance of different algorithms summarized in Table I. We assume Poisson traffic with symmetric arrival rates $\lambda_{i}=\lambda$, parameters $V_{i}=V$ and transmission rates $\mu_{i}^{\max }(t)=\mu^{\max }, i=1,2$. The achievable costs are shown in Figures 2 and 3 for $\mu^{\max }=5$ and for $V=$ 10 and 25, respectively. As expected, the centralized case outperforms the individually optimal solutions. The average costs are high for low arrival rates, since the possibility of performing network coding is lower than the case with higher loads, where it is more likely that queues are backlogged and it is possible to perform network coding. As expected, the average cost is reduced, as the parameter $V$ increases.

Figures 4 and 5 depict the average delay as function of the average cost per packet for different values of $V$. For the centralized optimal problem, the usual energy-delay trade-off is observed (such that the energy cost decreases, as delay increases), whereas the individual optimization problems may deviate from this behavior because sources do not have full information on each other's queue backlogs. 
TABLE I

SUMMARY OF THE PROPOSED COST SHARING ALGORITHMS

\begin{tabular}{|c|c|}
\hline A1 & Centralized optimal algorithm \\
A2 & Individually optimal algorithm \\
A3 & Worst-case response algorithm \\
A4 & Best-case response algorithm \\
A5 & Dynamic threshold-based algorithm \\
& with initial transmission sensing \\
A6 & Dynamic threshold-based algorithm \\
& with 1-bit queue backlog info \\
A7 & Algorithm w/ local queue info only, \\
& $T=\mu_{\max }, p_{i}=1, i=1,2$ \\
A7' & Algorithm w/ local queue info only, \\
& $T=\frac{\mu_{\max }}{p_{i}}$ for varying $p_{i}, i=1,2$ \\
A8 & Algorithm w/ local queue info only \\
& and w/o threshold, $p_{i}=1, i=1,2$ \\
\hline
\end{tabular}

Note that the average cost per packet achieved by (A6) is very close to the centralized algorithm (A1) and the delay is reduced while achieving the near-optimal cost. This effect is due to (A6)'s property of having a lower threshold, which causes sources to transmit without having to exceed the original threshold $V$ (whenever both source queues are backlogged) such that the average delay is reduced.

Figures 6 and 7 show the performance of algorithms without queue backlog information. Algorithm $\left(\mathbf{A} \mathbf{7}^{\prime}\right)$ is a variant of (A7), where threshold $T$ is $\frac{\mu_{\max }}{p_{i}}$. This illustrates the effect of dynamic threshold based on the transmission decision. If there is no information available regarding the queue backlog of the opponent source, and the threshold is greater than or equal to $\mu_{\max }$, the delay-optimal strategy is to select the lowest threshold $\mu_{\max }$, and $p_{i}=1$, i.e., each source transmits whenever its queue backlog exceeds the given threshold. Note that the cost performance is in accordance with (21). Operating without threshold decreases the cost for the case without queue backlog information. Any increase in the transmission probability overcomes the negative effect of the queue backlog mismatch on the average cost. Yet, the average cost of such an algorithm is significantly higher than algorithm (A6) with one bit queue state information, and it cannot approach the optimal value, unless the operating regime is very close to queue saturation.

\section{CONCLUSion}

In this paper, we considered the problem of minimizing the cost at a relay node that exchanges the incoming packets by network coding or routing depending on the availability of randomly arriving packets at both sources. The cost is shared by the sources depending on their rate allocation over the relay. We considered different levels of source cooperation and availability of queue state information at the sources. First, we derived the centralized control scheme to jointly optimize the cost and stable throughput rates. For distributed operation, sources share the cost of network coding for their

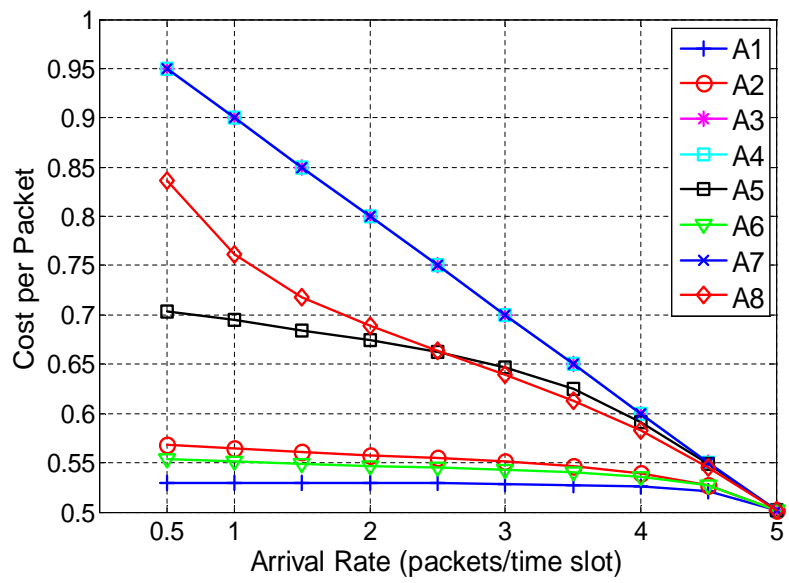

Fig. 2. Cost per packet as function of common arrival rate for $V=10$.

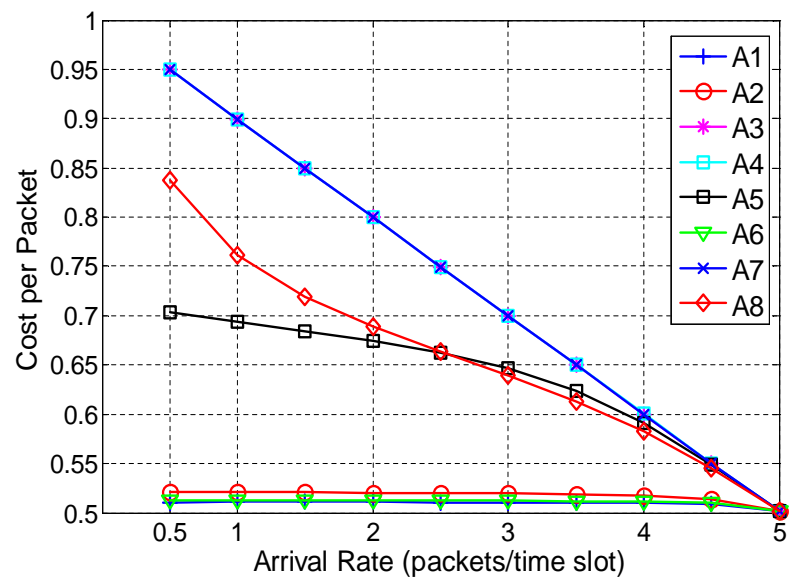

Fig. 3. Cost per packet as function of common arrival rate for $V=25$.

packets, whereas the residual cost for plain routing is charged to the individual source with higher rate requirement.

If sources are selfish with the individual cost objectives, cost sharing with network coding couples them in a rate allocation game. We derived the Nash equilibrium strategies and evaluate the non-cooperative cost-delay trade-offs. Then, we showed that pricing at the relay for the worst-case response can achieve the cost-optimal operation.

We also relaxed the assumption of queue state knowledge and considered simple rate allocation schemes, where sources either have one bit or no information regarding the queue state of the other source. With only one bit queue information, the threshold-based algorithms approach the optimal cost, whereas the packet delay does not not increase with the threshold as fast as the centralized cost-optimal scheme does. This leads to new cost-delay trade-offs for network coding.

Future work should generalize the model to arbitrary rate regions. This may further increase the coupling of the source queues by imposing joint constraints on the achievable rates. It is also crucial to extend the analysis of the cost-delay trade-offs to an arbitrary number of sources communicating through the relay node. This would require more complicated 


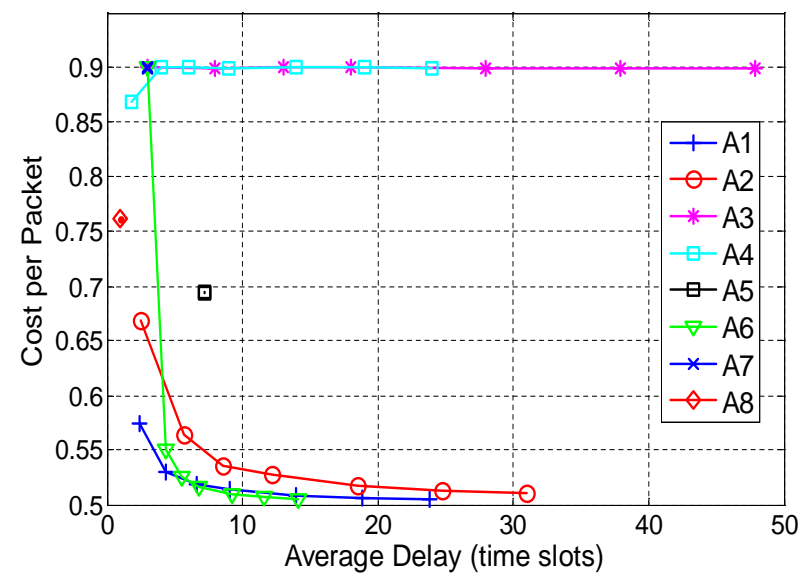

Fig. 4. Cost per packet as function of packet delay, $\lambda=1, \mu_{\max }=5$.

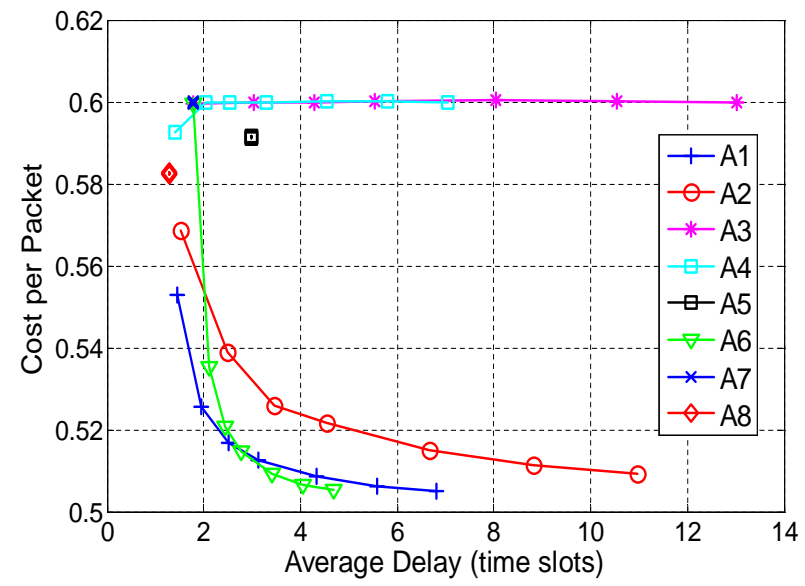

Fig. 5. Cost per packet as function of packet delay, $\lambda=4, \mu_{\max }=5$.

scheme of network coding (beyond pairwise packet matching) depending on the instantaneous backlogs of all sources.

\section{REFERENCES}

[1] R. Ahlswede, N. Cai, S. Y. R. Li, and R. W. Yeung. Network Information Flow. IEEE Transactions on Information Theory, 46(4):12041216, April 2000.

[2] S. J. Kim, P. Mitran, and V. Tarokh. Performance Bounds for Bi-directional Coded Cooperation Protocols. IEEE Transactions on Information Theory, 54(11):5235-5240, November 2008.

[3] S. Katti, I. Maric, A. Goldsmith, D. Katabi, and M. Medard. Joint Relaying and Network Coding in Wireless Networks. In Proc. of IEEE International Symposium on Information Theory, Nice, France, June 2007.

[4] D. S. Lun, N. Ratnakar, M. Medard, R. Koetter, D. R. Karger, T. Ho, E. Ahmed, and F. Zhao. Minimum-cost Multicast over Coded Packet Networks. IEEE Transactions on Information Theory, 52(6):26082623, June 2006.

[5] Y. Wu, P. A. Chou, and S.-Y. Kung. Minimum-energy Multicast in Mobile Ad Hoc Networks using Network Coding. IEEE Transactions on Communications, 53(11):1906-1918, November 2005.

[6] Y. E. Sagduyu and A. Ephremides. Cross-layer Optimization of MAC and Network Coding in Wireless Queueing Tandem Networks. IEEE Transactions on Information Theory, 54(2):554-571, February 2007.

[7] X. He and A. Yener. On the Energy Delay Trade-off of a Two-Way Relay Network. In Proc. of the Conference on Information Sciences and Systems, 2008, Princeton, NJ, March 2008.

[8] L. Tassiulas and A. Ephremides. Stability Properties of Constrained Queuing Systems and Scheduling Properties for Maximum Throughput in Multihop Radio Networks. IEEE Transactions on Automatic Control, 37(12):1936-1948, 1992.

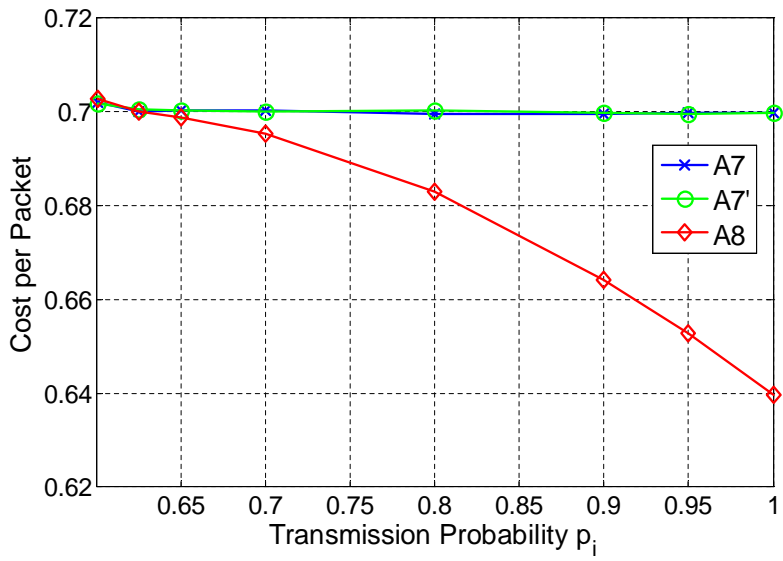

Fig. 6. Cost for varying transmission probability, $\lambda=3, \mu_{\max }=5$.

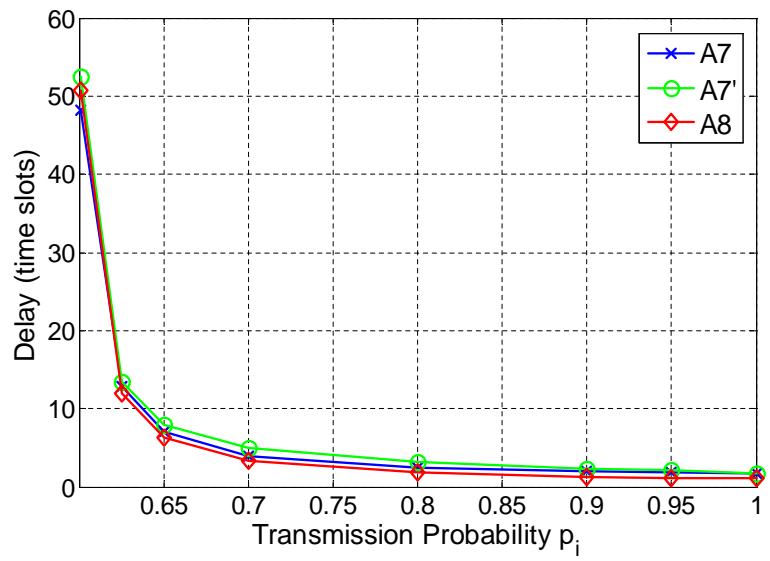

Fig. 7. Delay for varying transmission probability, $\lambda=3, \mu_{\max }=5$.

[9] M. J. Neely. Optimal Energy and Delay Tradeoffs for Multi-User Wireless Downlinks. IEEE Transactions on Information Theory, 53(9), September 2007.

[10] M. J. Neely. Energy Optimal Control for Time Varying Wireless Networks. IEEE Transactions on Information Theory, 52(7):29152934, July 2006.

[11] E. N. Ciftcioglu, A. Yener, and R. Berry. Stability Regions for TwoWay Relaying with Network Coding. In Proc. of Wireless Internet Conference, Maui, HI, November 2008.

[12] C. H. Liu and F. Xue. Network Coding for Two-way Relaying: Achievable Rate Regions, Sum Rate and Opportunistic Scheduling. In Proc. of IEEE International Conference on Communications, Beijing, China, May 2008.

[13] A. Eryilmaz and D. S. Lun. Control for Inter-session Network Coding. In Proc. of NETCOD, San Diego, CA, January 2007.

[14] T. Ho and H. Viswanathan. Dynamic Algorithms for Multicast with Intra-session Network Coding. IEEE Transactions on Information Theory, 55(2):797-815, February 2009.

[15] Y. E. Sagduyu, D. Guo, and R. Berry. Throughput Optimal Control for Relay-Assisted Wireless Broadcast with Network Coding. In Proc. of IEEE International Workshop on Wireless Network Coding, San Francisco, CA, June 2008.

[16] R. Berry and R. G. Gallager. Communication over Fading Channels with Delay Constraints. IEEE Transcations on Information Theory, 48(5):1135-1149, May 2002

[17] J. Price and T. Javidi. Network Coding Games with Unicast Flows. IEEE Journal on Selected Areas in Communications, 26(7):13021316, September 2008 .

[18] J. R. Marden and M. Effros. A Game Theoretic Approach to Network Coding. In Proc. of IEEE Information Theory Workshop on Networking and Information Theory, Volos, Greece, June 2009.

[19] D. Bertsekas and R. Gallager. Data Networks, 2nd edition. New Jersey: Prentice Hall, June 1992. 